\title{
Web Service Based Integration of Biological Interaction Databases
}

\author{
Seong Joon Yoo ${ }^{1}$, Min Kyung Kim² ${ }^{2}$, and Seon Hee Park ${ }^{3}$ \\ ${ }^{1}$ School of Computer Engineering, Sejong University, \\ 98 Gunja, Gwangjin, Seoul, Korea 143-747 \\ sjyoo@sejong.ac.kr \\ ${ }^{2}$ Department of Computer Science and Engineering, Ewha University, \\ 11-1 Daehyun-dong, Seodaemun-gu, Seoul, 120-750, Korea \\ minkykim@ewha.ac.kr \\ ${ }^{3}$ Bioinformatics Team, Electronics and Telecommunication Research Institute, \\ 161 Gajeong-Dong, Yuseong-Gu, Daejun, 305-350, Korea \\ shp@etri.re.kr
}

\begin{abstract}
While designing a web service based integration framework, it is not easy but most important to define API for biological SOAP servers. Therefore, we propose in this paper a web service API especially for the interaction databases: BIND, DIP and MINT. Each biological laboratory can configure their own application systems accessing these three databases transparently. The three databases are mirrored in our local computers on which we have implemented a prototype of SOAP servers for the interaction databases.
\end{abstract}

\section{Introduction}

As a part of efforts to solve bio-database integration problem, web services have been deployed in a few biological databases including myGRID[1], BioMoby[2], and KEGG[3]. myGRID is designed for data or service providers who want to build applications for biologist. None of these databases, however, provide web services for interaction information.

Even though the web services have much advantages, this technology is not rapidly introduced into biological field since it is not easy for computer engineers to define practically useful API due to their lack of biological information. System designers should study and know well about the application area - interaction databases in this work - to produce well-designed SOAP objects. We define API for the SOAP servers of interaction databases. The API is practically useful and well designed since it is based on the cooperation between biologists and computer engineers, and the experiences obtained from a datawarehouse design for the interaction databases in the past years. In this paper, we describe the interaction databases that have been spotlighted in terms of its data amount and importance thanks to the introduction of proteomics.

\section{Design of the Web Service API for the Interaction Databases}

Fig. 1 shows the web service based architecture of the interaction databases and examples of building application systems that are capable of accessing and integrating the three interaction databases. Each interaction database provides web service API with which client systems can implement bioinformatics systems for their own pur- 
poses. The BIND database[1] has three API classes: BindInteractionIF, BindPathwayIF, and BindComplexIF. The DIP interaction database[5] has defined a single class: DIPInteractionIF. The MINT interaction database[6] provides one class: MINTInteractionIF.

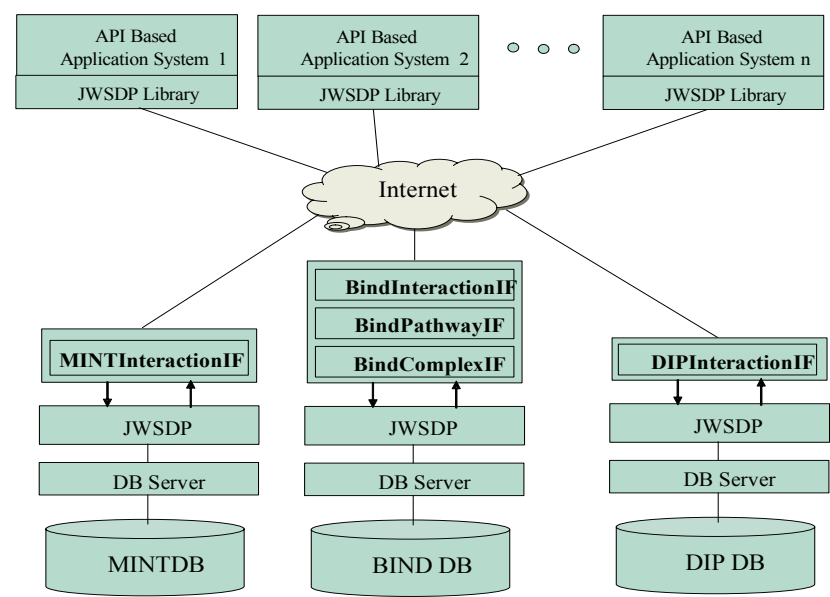

Fig. 1. The Architecture of the Integrated Interaction Database with Web Services

\section{An Application Example}

Fig. 2 show an example of using the API defined in the previous section for accessing pathways that include a query interaction from the BIND database. Fig. 2 is a SOAP message requesting a pathway ID including the given interaction ID. We have developed a prototype of the SOAP servers and databases in our local systems. Since original sites of the above three interaction databases do not provide their own SOAP servers, we needed to build our own databases by copying the original data in nonrelational database format and transforming these into relational database format. We have used three servers with Intel Pentium4 1.9 CPU, Memory 512MB, and the software environment of Windows 2000 Professional, JWSDP 1.3, and Java 1.4.

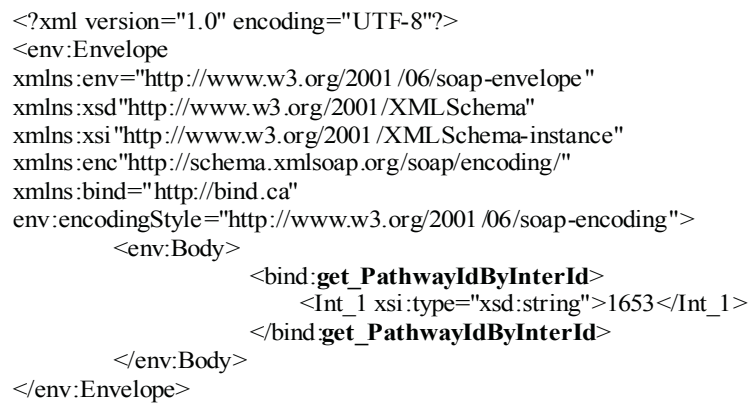

Fig. 2. A SOAP Message Requesting a Pathway ID Including the Given Interaction ID 


\section{Conclusion}

In this paper, we have proposed a practically useful API for SOAP servers of interaction databases. Bioinformaticians may build their own client software that accesses data from those three interaction databases through these objects on the SOAP servers. Once the source databases implement the proposed API on their own databases and SOAP servers, worldwide users can access the interaction databases more easily. Users can use this system with the interaction of gene ontology for semantic integration of interaction databases. We are working on this issue.

\section{References}

1. Stevens RD, Robinson AJ, and Goble CA.: myGrid: personalised bioinformatics on the information grid, Bioinformatics. 19, 2003.

2. Wilkinson, M.D. and Links, M.: BioMOBY: an open source biological web services proposal, Brief Bioinform. 3, 2002.

3. Kawashima, S., Katayama, T., Sato, Y. and Kanehisa, M.: A Web Service Using SOAP, WSDL to Access the KEGG System, Genome Informatics, 14, 673-674, 2003

4. Bader, G.D. and Hogue, C.W.: BIND-a data specification for storing and describing biomolecular interactions, molecular complexes and pathways, Bioinformatics 16, 465-477, March 2000.

5. http://dip.doe-mbi.ucla.edu

6. Cesareni, G. and Gimona, M.: MINT: a Molecular INTeraction database, A. Zanzoni et al./FEBS Letters 513, 135-140, December 2001. 